\title{
Wunderlich syndrome: spontaneous atraumatic rupture of the kidney
}

\begin{abstract}
Atraumatic spontaneous retroperitoneal haemorrhage is a distinct clinical entity with potentially life-threatening complications. The commonest aetologies include ruptured aortic or visceral aneurysm, spontaneous rupture of the kidney and patients with coagulopathies. Spontaneous rupture of the kidney can occur due to underlying renal pathologies such as malignancy, angiomyolipoma, vascular malformation, vasculitis and infection. Approximately $5 \%$ of such cases occur without an identifiable renal pathology and are termed as 'idiopathic'. Idiopathic spontaneous rupture has been described among patients on chronic haemodialysis associated with acquired cystic disease of the kidney. We describe the extremely rare instance of a patient with a failed renal allograft and recently started on haemodialysis who suffered an idiopathic spontaneous rupture of his native kidney.
\end{abstract}

\section{Keywords: Spontaneous renal rupture, chronic haemodialysis, retroperitoneal haemorrhage, renal tumour}

\section{Introduction}

Atraumatic Spontaneous Rupture of the Kidney (ASRK) is a rare but definite clinical entity that can often lead to diagnostic and management dilemma. It is often associated with an underlying renal pathology such as malignancy, angiomyolipoma, infection, renal cysts or arterio-venous malformation [1,2]. The resulting retroperitoneal haemorrhage can often lead to life-threatening blood loss and haemodynamic instability. A high degree of clinical suspicion coupled with cross-sectional imaging is the cornerstones in diagnosis, enabling prompt intervention. While the great majority of such patients require surgical exploration, minor degree rupture with limited internal bleeding can be managed with conservative measures. We report a case of ASRK in a 'morphologically normal' native kidney of a patient who had been recently initiated on haemodialysis following a previously failed renal allograft.

The Case The patient was a 48-year-old male who had undergone a live donor renal transplantation for IgA nephropathy 3 years before. After an initial period of excellent graft function, he suffered from allograft failure due to chronic allograft nephropathy. His graft function was excellent until two months prior to the current presentation, when he presented with proteinuria, edema and rising serum creatinine. Ultrasonography revealed a morphologically normal allograft. The native kidneys were small and contracted, measuring $7.1 \mathrm{~cm}$ (right) and $7.2 \mathrm{~cm}$ (left), with no focal lesions, cysts or calculi. An allograft biopsy was performed and demonstrated a mixed picture of T-cell and antibody mediated rejection with features of chronic calcineurin toxicity. He was treated with methylprednisolone pulses followed by therapeutic plasma exchange for 12 days. Response to treatment was poor and the patient ended up with graft failure whereby he was re-initiated on regular haemodialysis 4 weeks prior to the index presentation. He was maintained on baseline immunosuppression (prednisolone and tacrolimus) with an aim of re-transplantation from his wife who was worked up as a live donor.

On this admission, he presented to the hospital emergency department with sudden onset, severe, colicky left-sided loin pain. He denied fever or a history of trauma. He had undergone his scheduled haemodilaysis the day before. He was not on any other long-
Nalaka Gunawansa*, Dinesha Sudusinghe, Dilushi Wijayaratne and Chathurika Beligaswatta

National Institute of Nephrology, Dialysis and Transplantation-Colombo, Sri Lanka

*Author for correspondence:

vascular@drnalakagunawansa.com 
term anticoagulants or antiplatelet therapy. On examination he was pale with a pulse rate and blood pressure of 92 beats per minute and $140 / 80 \mathrm{mmHg}$ respectively. His hemoglobin was $5.4 \mathrm{~g} / \mathrm{dL}$.

Ultrasonography revealed a heterogeneous echogenic area in the left native renal bed, measuring $7 \times 9 \times 18 \mathrm{~cm}$ extending along the psoas muscle, suggestive of a hematoma. This was confirmed by Computerized Tomography (CT) scan (FIGURE 1). The left kidney could not be identified separately from the haematoma while there was no evidence of renal malignancy, cystic disease, calculi, hydroureter or hydronephrosis. $\mathrm{He}$ was resuscitated and commenced on empirical intravenous antibiotics; meropenem and teicoplanin.

The same evening, the patient had a further drop in hemoglobin $(3.1 \mathrm{~g} / \mathrm{dL})$ and required transfusion of seven units of leuco-depleted red cell concentrate for stabilization. At this time, a decision was taken for surgical exploration. The surgical approach was through an extended $12^{\text {th }}$ rib trans-costal incision. A large retroperitoneal hematoma was found with complete disruption of the renal parenchyma along its entire length (FIGURE 2). There was no demonstrable

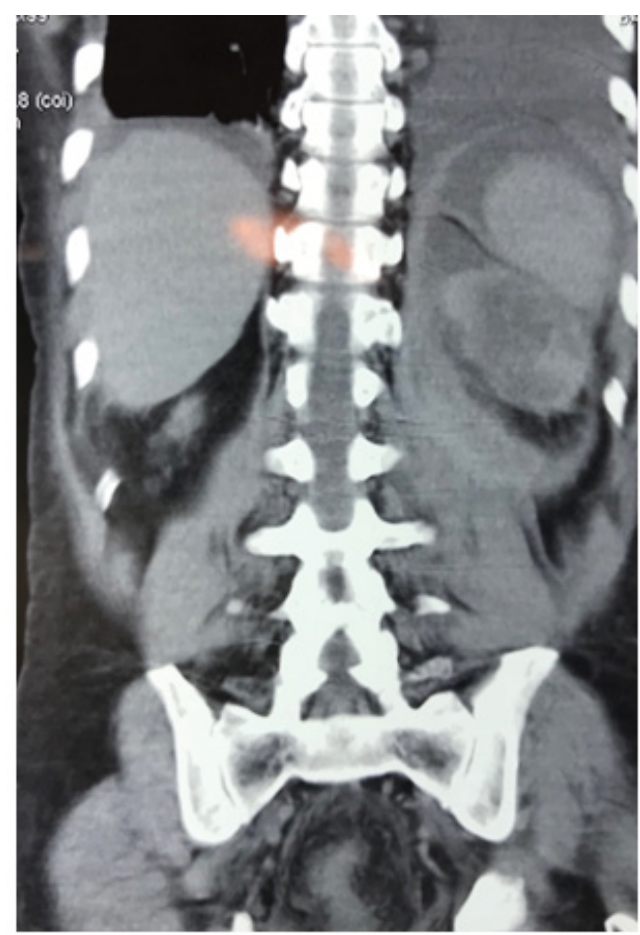

FIGURE 1: CT image (coronal plane) of the ruptured left native kidney and retroperitoneal haematoma concealed in the renal capsule. cystic or tumour tissue intra-operatively. The renal pedicle was clamped away from the hilum close to the aorta and the immediate bleeding was controlled. Haemodynamic stability was achieved with fluid and blood product replacement. The ruptured kidney with the amorphous large haematoma was evacuated before achieving meticulous haemostasis (FIGURE 3). The operative field was washed with sterile water and closed with a drain in the native renal bed.

The post-operative course was uneventful, and he maintained stable haemodynamic parameters while the post-operative haemogloin level remained around $9 \mathrm{~g} / \mathrm{dL}$. The surgical drain was removed on day-02 while intravenous

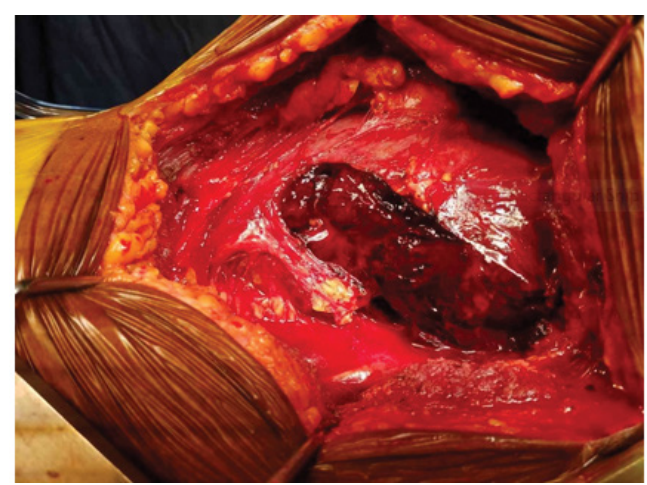

FIGURE 2: Trans-costal (12th rib) incision to access the retroperitoneum exposing the large haematoma and ruptured left kidney.

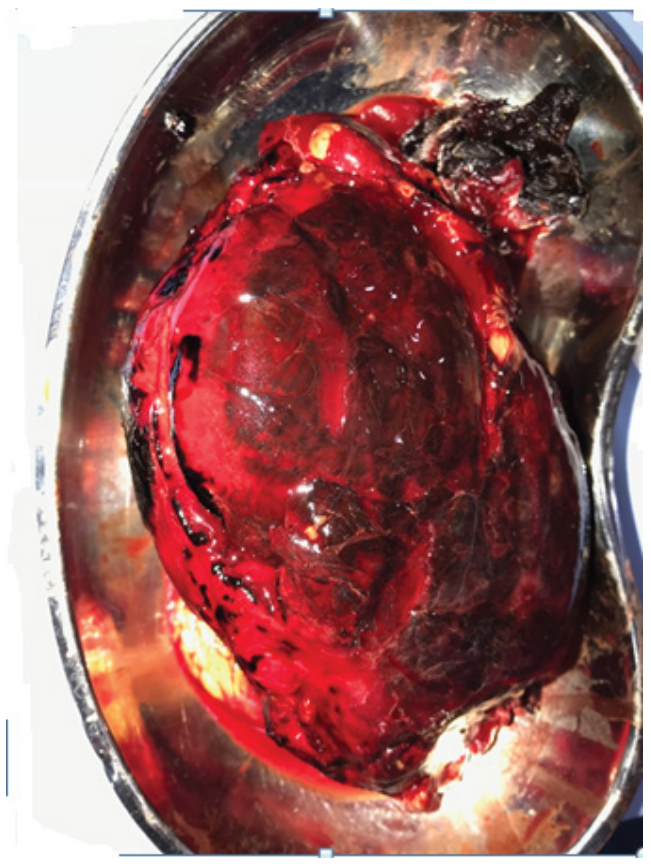

FIGURE 3: Removed specimen of the ruptured kidney and contained haematoma being sent for histological assessment. 
antibiotics were continued till day-05. Maintenance regular haemodialysis was continued in the post-operative period.

Histology of the specimen confirmed rupture of the native kidney with no evidence of infection, cyst or tumour. Approximately 12 weeks after complete recovery, he underwent successful re-transplant from his wife. The new allograft was placed in the left iliac fossa and he remains with excellent graft function at the time of writing ( 28 weeks post re-transplant).

\section{Discussion}

ASRK was first described by Bonet (1679), although its scientific significance was made famous by Wunderlich in his description in 1856 [3]. The classical clinical presentation consists of the Lenk's triad of flank pain, palpable loin mass and hypovolaemia secondary to internal bleeding. Rupture of the kidney may involve either the collecting system or parenchyma. Parenchymal rupture results in severe bleeding with either subcapsular or perirenal haematoma.

The largest series of ASRK was described by Zhang and colleagues (2002) consisting of 165 patients [4]. The commonest underlying aetiology after histological assessment was tumour (61\%). Other aetiologies included vascular malformations (17\%) and infections (04\%), while $6.7 \%$ were declared as truly idiopathic without any identifiable lesion as in our index patient. The same series described a slightly higher male preponderance with a male to female ratio of $6: 5$ and a mean age of 46.8 years (range 4 months- 89 years).

ASRK among patients on haemodialysis has been described in several publications including the report by Bensalah and colleagues (2003) [5,6]. Levine et al. have also described ASRK in the native kidneys of patients who had undergone renal transplantation [7]. This distinct clinical entity is often attributed to acquired renal cystic disease seen with chronic haemodialysis. Acquired cystic disease has been described in up to $95 \%$ of patients on chronic hameodialysis. However, our index patient had no prior history of cystic disease and haemodialysis was started only 4 weeks prior to the current presentation. Furthermore, imaging of both kidneys and subsequent histology also did not show any evidence of cystic disease.
The diagnosis of spontaneous retroperitoneal bleeding requires a high degree of clinical suspicion based on the classical presentation and an unexplained drop in haemoglobin as seen in the index patient. The differential diagnosis includes rupture of abdominal aortic aneurysm, visceral aneurysms or other visceral bleeding such as in ASKR. Confirmation of diagnosis requires definitive imaging. Although easily accessible and non-invasive, the diagnostic accuracy of ultra-sound (US) scan in retroperitoneal haemorrhage has been documented around 56\% (7). In contrast, the diagnostic accuracy of CT scan is $>99 \%$. CT cross-sectional imaging also allows to exclude pathology in the contralateral kidney as well as other retroperitoneal structures such as the abdominal aorta. Considering the commonest aetiology of ASRK is underlying renal tumour, the diagnostic sensitivity of detecting a renal tumour in the background of ASRK on US and CT are $11 \%$ and $57 \%$ respectively (7). Nevertheless, with the advent of more advanced high-resolution CT technology, the current yield rate of small renal cell carcinoma on CT is much higher (>87\%) [4.8]. Magnetic Resonance Imaging (MRI) may be useful in patients where impaired renal function precludes contrast CT imaging [9].

Management is according to the underlying etiology and hemodynamic status of the patient. In majority of cases severe haemorrhage necessitates open surgical intervention to control bleeding and nephrectomy. The possibility of underlying renal tumour and the poor sensitivity of pre-operative imaging to confirm the presence of tumour within the haematoma has been put forward as justification for radical nephrectomy in such patients. This view has been advocated by numerous authors including Bagley Kendall and Novicki in their respective series of ASRK due to the seemingly high probability of finding small renal cell carcinomas during histological assessment [10-12].

In patients who are haemodynamically stable and with no evidence of on-going blood loss, conservative non-operative management has also been described [13]. This approach has mainly been advocated for patients on chronic haemodialysis where the possibility of acquired renal cystic disease and bleeding in the cysts is the likely pathology. With current high-resolution CT imaging, while this avoids a major surgical 
intervention, it requires accurate and close CT surveillance performed at 3-6 month intervals to exclude the possibility of underlying renal tumour [9].

\section{Conclusion}

Spontaneous atraumatic retroperitoneal bleeding is a distinct clinical entity that requires a high degree of clinical suspicion and immediate intervention. Classical clinical presentation along with findings on imaging help in establishing the diagnosis. The possibility of ASRK should be considered along with other differential diagnoses especially in the younger patients. Cross-sectional CT imaging is considered the gold standard investigation in confirming the diagnosis as well as identifying possible underlying renal pathology. Renal tumours account for over $60 \%$ of all patients with ASRK. Haemodynamic instability and ongoing bleeding warrants mandatory surgical exploration and control along with radical nephrectomy. Even among the patients who attain haemodynamic stability with resuscitation, a low threshold should be maintained for surgical exploration and nephrectomy due to the high incidence of renal tumour.

Explicit informed written consent has been obtained from the patient to share these clinical data for purposes of medical education. 


\section{References}

Howalt J, Squires JW. Spontaneous rupture of the kidney. Am. J. Surg. 123, 484-488 (1972).

Wolff JM, Jung PK, Adam G, Jakse G. Spontaneous retroperitoneal haemorrhage associated with renal disease. J. R. Coll. Surg. Edinb. 43(1), 5356 (1998).

Wunderlich C. Handbuch der Pathologie und Therapie. Stuttgart: Ebner \& Seubert (1856).

Zhang JQ, Fielding JR, Zou KH. Etiology of spontaneous perirenal hemorrhage: a meta-analysis. J Urol. 167(4), 1593-1596 (2002).

Bensalah K, Martinez F, Ourahma S, Bitker MO, Richard F, Barrou B. Spontaneous rupture of non-tumoral kidneys in patients with end stage renal failure: Risks and management. Eur. Urol. 44(1), 111-114 (2003).

Malek-Marin T, Arenas D, Gil T, et al. Spontaneous retroperitoneal hemorrhage in dialysis: a presentation of 5 cases and review of the literature. Clin. Nephrol. (3), 229-244 (2010).

Levine E, Grantham JJ, MacDougall ML. Spontaneous subcapsular and perinephric hemorrhage in end-stage kidney disease: clinical and CT findings. AJR. Am. J. Roentgenol. 148(4), 755-758 (1987).

Marino G, Pedalino M, Di Primio OG, et al. Wunderlich Syndrome. Clinical and Therapeutic Aspects of a Long-Term Experience. Urol. J. 77(3), 193-197 (2010).

Albi G, Del Campo L, Tagarro D. Wünderlich's syndrome: Causes, diagnosis and radiological management.
Clin. Radiol. 57(9), 840-845 (2002).

Bagley DH, Feldman RA, Glazier W, Traurig A, Kraus P. Spontaneous Retroperitoneal Hemorrhage From Renal Carcinoma. JAMA J. Am. Med. Assoc. 248(6), 720-721 (1982).

Kendall AR, Senay BA, Coll ME. Spontaneous subcapsular renal hematoma: diagnosis and management $J$. Urol. 139(2), 246-250 (1988).

Novicki D, Turlington J, Ball TJ. The evaluation and management of spontaneous perirenal hemorrhage. PubMed - NCBI. J. Urol. 123(5), 764765 (1980).

Koo V, Duggan B, Lennon G. Spontaneous rupture of kidney with peri-renal haematoma: A conservative approach. Ulster. Med. J. 73(1), 53-56 (2004). 